\title{
Spinosin Attenuates Osteoclastogenesis Through Suppressing NF-KB by the Activation of Nrf2/HO-1 Signaling in Osteoporosis
}

Bo Liu

Linyi Central Hospital

Yuna Zhang ( $\nabla$ YunaZhangLinyi@163.com )

Linyi Central Hospital https://orcid.org/0000-0002-4100-5161

\section{Research}

Keywords: osteoporosis, osteoclastogenesis, Spinosin, Nrf2/HO-1 signaling, NF-KB

Posted Date: January 29th, 2021

DOl: https://doi.org/10.21203/rs.3.rs-154482/v1

License: (c) (i) This work is licensed under a Creative Commons Attribution 4.0 International License.

Read Full License 


\section{Abstract}

Objective

Osteoporosis is a prevalent metabolic skeletal disorder featured by microarchitecture bone injury and excessive osteoclastic activity.Here, we aimed to explore the effect of Spinosin on osteoclastogenesis of osteoporosis.

Design

The receptor activator of nuclear factor-kappaB ligand (RANKL)-induced osteoclastogenesis model was established in bone marrow macrophages (BMMs) in vitro. The ovariectomy (OVX)-induced bone loss mouse model was constructed in vivo, followed by micro-CT analysis, Histomorphometric analysis, Hematoxylin and Eosin (H\&E) and TRAP staining.

Results

Our data showed that the treatment of Spinosin significantly inhibited the TRAP positive osteoclast and bone resorption induced by RANKL in the BMMs. Spinosin significantly reduced the expression of osteoclast-specific factors, including osteoclast stimulatory transmembrane protein (OC-STAMP), dendritic cell-specific transmembrane protein (DC-STAMP), cathepsin K (CTSK), TRAP, c-Fos and nuclear

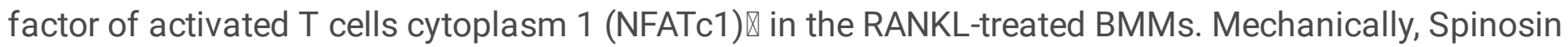
was able to inactivate NF-KB by stimulating Nrf2/HO-1 signaling in BMMs. The trabecular space (Tb.Sp), trabecular number (Tb.N), trabecular thickness (Tb.Th), and bone volume to total volume (BV/TV) were inhibited by OVX treatment, and Spinosin could reverse the effect in the bone resorption mouse model. The OVX-induced serum levels of tumor necrosis factor-a (TNF-a) and tartrate-resistant acid phosphatase 5 B (TRAcp5B) were blocked by Spinosin in the mice. Moreover, Spinosin was able to alleviate OVXinduced loss of femur bone and osteoclasts in vivo.

\section{Conclusions}

In conclusion, Spinosin attenuates osteoclastogenesis of osteoporosis through inhibiting NF-KB by activating Nrf2/HO-1 expression. Spinosin may serve as the potential candidate for the treatment of osteoporosis.

\section{Introduction}

Osteoporosis serves as a prevalent skeletal disorder featured by the weak bone magnitude and microarchitecture bone injury, affecting 10 of thousands of post-menopausal women because of estrogen insufficiency $(1,2)$. A recent report shows that 65-years men and 55-years women and older possess a heightened fracture chance affected by osteoporosis (3). Osteoporosis fracture is correlated with enhanced death and cause an enormous financial burden of modern people and society (4), yet currently, rare clinical medications are accessible for osteoporosis (5-7). In recent years, targeting 
osteoclastogenesis becomes an available healing approach to osteoporosis $(8,9)$. However, the popular anti-osteoporosis therapies, such as bisphosphate and denosumab, accompany the jaw necrosis risk, leading to application limitation $(10,11)$. Therefore, innovative alternative treatment candidates for osteoporosis that are able to regulate osteoclastogenesis through various mechanisms with advanced risk-benefit outlines are necessarily required.

Traditional Chinese herbs have been applied thousands of years for the treatment of human diseases and have drawn growing attention in drug discovery investigation in recent years (12). Suanzaoren, also termed Semen Ziziphi Spinosae (SZS), is the Ziziphus jujuba Mill (13). Usually, SZS serves as a hypnoticsedative plant, and Asian medication practitioners generally utilize it to prescribe anxiety and insomnia (14). SZS includes more than fifty active composites, such as C-glycoside flavonoids, cyclopeptide alkaloids, and saponins (15). Among them, a C-glycoside flavonoid called 2"--3-0-glucopyranosyl swertisin (Spinosin) is applied in Western countries as healthcare agents and exhibits biomedical activities, including hypnotic and anxiolytic impacts (16). Besides, Spinosin has been identified to enhance mice's cognitive accomplishment, attenuate memory deficiencies, and improve neurogenesis $(17,18)$. Recent research indicates that Spinosin displays neuroprotective influences in Alzheimer's disease mouse model (19). Moreover, Spinosin is able to stimulate Nrf2/HO-1 signaling to inhibit the aggregation and production of $A \beta_{1-42}(20)$. Spinosin may present a potential role in the modulation of osteoporosis due to that Nrf2/HO-1 signaling performs a important funcion during osteoclastogenesis.

The development of osteoporosis and osteoclastogenesis is complicated and many crucial signaling pathways are involved in the processes. Oxidative stress is a crucial process correlated with osteoporosis's pathogenesis, and the oxidative stress progression-induced modulation of the $\mathrm{Nrf2} / \mathrm{HO}-$ $1 / \mathrm{NF}-\mathrm{KB}$ (p65) signaling has been extensively investigated in osteoporosis as well (21). More and more evidence shows that the accumulation of reactive oxygen species (ROS) causes oxidative stress at condition of medicine stimulation, illnesses, or aging, and followed by inhibiting the stimulation of Nrf/HO-1 expression and enhancing p65, leading to the progression and genesis of osteoporosis $(22,23)$. Accordingly, the agents that is able to activate Nrf/HO-1 signaling and thereby suppress NF-KB signaling may be the agents for the osteoporosis therapy.

In this study, we were interested in the role of Spinosin in the pathogenesis of osteoporosis. We identified that Spinosin could inhibit RANKL-induced osteoclastogenesis in vitro. Spinosin was able to attenuate OVX-induced loss of femur bone and osteoclasts in vivo. Melancholically, Spinosin inhibiting NF-KB by enhancing Nrf2 expression. We demonstrated a novel function of Spinosin in alleviating osteoclastogenesis of osteoporosis by suppressing NF-KB through activating Nrf2/HO-1 signaling.

\section{Materials And Methods}

\subsection{The isolation of bone marrow macrophages (BMMs)}


BMMs were used to obtain the osteoclast progenitor cells. Shortly, BMMs were obtained from 8-10-weekold C57 mice's femurs by flushing the MEM (Gibco, USA) medium's bone marrow cavity. BMMs were cultured in MEM medium with 10\% FBS-a-MEM (Gibco, USA). The unattached BMMs were gathered and cultured for three days in the MEM medium with M-CSF $(25 \mathrm{ng} / \mathrm{mL})$ and FBS $(10 \%)$ to settle BMMs

\subsection{Osteoclast differentiation}

For the osteoclast differentiation, BMMs were collected in a-MEM medium with M-CSF $(25 \mathrm{ng} / \mathrm{mL})$, RANKL (100 ng/mL), and FBS (10\%). The MC3T3-E1 cells were cultured in the a-MEM medium (Gibco, USA) at $37^{\circ} \mathrm{C}$ with $5 \% \mathrm{CO}_{2}$. For osteoblast differentiation, the cells were plated into the 24-well wells and incubated for 7 days or 21 days in the a-MEM comprising 10\% FBS, ascorbic acid ( $50 \mu \mathrm{g} / \mathrm{ml}$, Sigma, USA). The osteoblast differentiation was analyzed by ALP staining and alizarin red staining, respectively. The siRNA of Nrf2 (5'-GUAAGAAGCCAGAUGUUAAdUdU-3') was obtained (RiboBio, China) The transfection in the cells was performed by Liposome 3000 (Invitrogen, USA) according to the manufacturer's instructions.

\subsection{CCK-8 assay}

The cell viability was analyzed by the CCK-8 assays. About $5 \times 10^{3}$ BMMs were plated in 96 -well paltes and incubated for 12 hours, followed by indicated treatment. The BMMs were incubated with a CCK-8 solution (KeyGEN Biotech, China) and culture for another 2 hours at $37^{\circ} \mathrm{C}$. The cell viability was analyzed at 450nm absorbance by applying the ELISA browser (Bio-Tek EL 800, USA).

\subsection{Tartrate-resistant acid phosphatase (TRAP) staining}

Cells were cleaned using PBS and fixed for 5 minutes based on the paraformaldehyde. Cells were cultured at $37^{\circ} \mathrm{C}$ for 1 hour away from light in the reaction from Leukocyte Acid Phosphatase Assay Kit (Sigma) according to the manufacturer's guidance. Cells were distilled by water, and TRAP-positive cells comprising five or more nuclear were imagined by microscopy and calculated as mature osteoclasts. The quantification was performed by applying the ImageJ software.

\subsection{Bone resorption assay}

The bone resorption was analyzed by applying a pit formation assay. BMMs were palted in the 24-well plates. The cells were re-collected every three days with fresh a-MEM medium. After 5 days, the cells were treated with $\mathrm{NH}_{4} \mathrm{OH}(1 \mathrm{~N}, 5$ minutes) to eliminate the attached BMMs. The bone resorption area was quantified by utilizing the image $J$ software.

\subsection{Quantitative reverse transcription-PCR (qRT-PCR)}

The total RNAs were extracted by TRIZOL (Invitrogen, USA) from the tissues and cells. The first-strand cDNA was synthesized using Stand cDNA Synthesis Kit (Thermo, USA) as the manufacturer's instruction. The qRT-PCR was carried out by applying SYBR Real-time PCR I kit (Takara, Japan). The standard control for mRNA was GAPDH. Quantitative determination of the RNA levels was conducted by SYBR 
GreenPremix Ex TaqTM II Kit (TaKaRa, Japan). The experiments were independently repeated at least three times. The primer sequences are as follows:

DC-STAMP Forward: 5'-AAAACCCTTGGGCTGTTCTT-3',

DC-STAMP Reverse: 5'-AATCATGGACGACTCCTTGG-3'

c-Fos Forward: 5'- CGGGTTTCAACGCCGACTA-3'

c-Fos Reverse: 5'- TGGCACTAGAGACGGACAGAT-3'

OC-STAMP Forward: 5'- CTGTAACGAACTACTGACCCAGC-3'

OC-STAMP Reverse: 5'- CCAGGCTTAGGAAGACGAAGA-3'

NFATc1 Forward: 5'-CCGTTGCTTCCAGAAAATAACA-3'

NFATc1 Reverse: 5'-TGTGGGATGTGAACTCGGAA-3'

TRAP Forward: 5'-CTGGAGTGCACGATGCCAGCGACA-3'

TRAP Reverse: 5'-TCCGTGCTCGGCGATGGACCAGA-3'

CTSK Forward: 5'-CTTCCAATACGTGCAGCAGA-3'

CTSK Reverse: 5'-TCTTCAGGGCTTTCTCGTTC-3'

\subsection{Western blot analysis}

Total proteins were extracted using RIPA buffer (Millipore, USA). Nuclear and cytoplasmic proteins were extracted by applying the Nuclear Extraction Kit (Thermo, USA) and Cytoplasmic Extraction Kit (Thermo, USA), respectively. Protein concentrations were measured by using the BCA Protein Quantification Kit (Abbkine, USA). Same concentration of protein was divided by SDS-PAGE (12\% polyacrylamide gels), transferred to PVDF membranes (Millipore, USA) in the subsequent step. The membranes were hindered with $5 \%$ milk and hatched overnight at $4^{\circ} \mathrm{C}$ with the primary antibodies for Nrf2 (1: 1000, CST, USA), HO-1 (1: 1000, Abcam, USA), IKBa (1: 1000, CST, USA), p65 (1: 1000, CST, USA), LaminB (1: 1000, CST, USA), NFATc1 (1: 1000, CST, USA), c-Fos (1: 1000, CST, USA), and $\beta$-actin (1: 1000, CST, USA), in which $\beta$-actin served as the control. Then, the corresponding second antibodies (1: 1000, Abcam, USA) were used for hatching the membranes 1 hour at room temperature, followed by the visualization by using an Odyssey CLx Infrared Imaging System.

\subsection{OVX-induced bone loss mouse model}

The C57BL/ 6 mice (8 weeks, male) were randomly divided in three groups $(n=5)$, including control group, $\mathrm{OVX}$ treatment group, and OVX + Spinosin co-treatment group. In the control group, the mice were injected equal volume water; In the OVX treatment group, the mice were intraperitoneally injected with 
streptozotocin (STZ, $50 \mathrm{mg} / \mathrm{kg}, 5$ days, sigma, USA); In the OVX + Spinosin co-treatment group, the mice were intraperitoneally injected with OVX and orally administered with Spinosin-treated group (50 $\mathrm{mg} / \mathrm{kg} / \mathrm{day}$ ). The mice were sacrificed after 9 day, and the tibias from the mice were subjected in highresolution micro-CT analysis and Histomorphometric analysis. The levels of TRAcp5B and TNF-a were measure by ELISA assays. The loss of femur bone and osteoclasts was analyzed by Hematoxylin and Eosin (H\&E) and TRAP staining. Animal care and method procedure were authorized by the Animal Ethics Committee of Linyi Central Hospital.

\subsection{Statistical analysis}

Data was presented as mean $\pm S D$, and the statistical analysis was conducted by GraphPad prism 7. The unpaired Student's $t$-test was used to compare two groups, and the one-way ANOVA was utilized to compare among multiple groups. $P<0.05$ were considered as statistically significant.

\section{Results}

\subsection{Spinosin inhibits RANKL-induced osteoclastogenesis}

To assess the potential cytotoxic effect of Spinosin (Fig. 1A) on BMMs, the BMMs were treated with Spinosin at the dose of 5, 10, 25, 50, 100, 200, and $400 \mu \mathrm{M}$. CCK-8 assay showed that Spinosin treatment $(\leq 100 \mu \mathrm{M})$ failed to affect the cell viability of BMMs compared with the $0 \mu \mathrm{M}$ Spinosin, suggesting that Spinosin has no cytotoxic effect on BMMs at dose up to $100 \mu \mathrm{M}$ (Fig. 1B). Then, we selected the dose of 25,50 , and $100 \mu \mathrm{M}$ for examining the role of Spinosin in the modulation of RANKL-induced osteoclast formation. Our data showed that the treatment of Spinosin significantly inhibited the TRAP $(+)$ osteoclasts in a dose-dependent manner in the RANKL-treated BMMs compared with the $0 \mu \mathrm{M}$ Spinosin $(P<0.001$, Fig. 1C). Meanwhile, the resorption pit formation assay revealed that Spinosin treatment dosedependently reduced RANKL-caused bone resorption compared with the $0 \mu \mathrm{M}$ Spinosin $(P<0.001$, Fig. 1D). Taken together, these data indicate that Spinosin can inhibit RANKL-induced osteoclastogenesis in vitro.

\subsection{Spinosin reduces the expression of osteoclast-specific genes in the RANKL-treated BMMs}

Then, to validate the effect of Spinosin on osteoclastogenesis, the expression of osteoclast-specific genes, including nuclear factor of activated T cells, cytoplasm 1 (NFATc1), c-Fos, TRAP, cathepsin K (CTSK), dendritic cell-specific transmembrane protein (DC-STAMP), and osteoclast stimulatory transmembrane protein (OC-STAMP), was analyzed by qPCR. Our data showed that the treatment of Spinosin significantly reduced the expression of NFATc1, c-Fos, TRAP, CTSK, DC-STAMP, and OC-STAMP in a dose-dependent manner in the RANKL-treated BMMs compared with the $0 \mu \mathrm{M}$ Spinosin $(P<0.001$, Fig. 2). Together, these results suggest that Spinosin inhibits the expression of osteoclast-specific genes in the RANKL-treated BMMs. 


\subsection{Spinosin fails to affect osteoblast differentiation.}

Then, we further explored the effect of Spinosin on osteoblastic differentiation. To this end, the MC3T3E1 cells were treated with Spinosin at indicated doses. CCK-8 assays showed that Spinosin did not affect the cell viability of MC3T3-E1 cells, suggesting that Spinosin has no cytotoxic effect on MC3T3-E1 cells $(P>0.05$, Fig. 3A). ALP staining and Alizarin Red staining revealed that the osteoblastic differentiation of MC3T3-E1 cells was not affected by Spinosin treatment (Fig. 3B). Meanwhile, the mRNA expression levels of osteoblast differentiation markers, such as amphiphysin-like protein 1 (ALP1), runt-related transcription factor 2 (RUNX2), and osteocalcin (OCN), were not chanced by Spinosin treatment in the cells $(P>0.05$, Fig. $3 C)$. Taken together, these results indicate that Spinosin fails to affect osteoblast differentiation.

\subsection{Spinosin suppresses NF-kB signaling by activating Nrf2 pathway}

Next, we further assessed the underlying mechanism of Spinosin-mediated osteoclastogenesis. Significantly, our data showed that the treatment of Spinosin enhanced the expression of nuclear factor E2-related factor 2 (Nrf2) and heme oxygenase-1 (HO-1) in the BMMS $(P<0.001$, Fig. 4A and B). Moreover, the treatment of RANKL remarkably decreased the cytoplasm expression of inhibitor of nuclear factor kappa B alpha ( $\mathrm{IKBa}$ ) and increased the nucleus accumulation of nuclear factor-kappa B (NF-KB, p65), in which the treatment of Spinosin could reverse the effect in the BMMs $(P<0.001$, Fig. $4 C$ and $D)$. Moreover, Western blot analysis demonstrated that the expression of nuclear Nrf2 and cytoplasm HO-1 was reduced, while the expression of nuclear p 65 was enhanced by the Nrf2 shRNA in the Spinosin and RANKL co-treated BMMs compared with control shRNA $(P<0.001$, Fig. $4 \mathrm{E}$ and $\mathrm{F})$. Together, these results suggest that Spinosin is able to suppresse NF-KB signaling by activating Nrf2 pathway

\subsection{Spinosin inhibits RANKL-induced expression of c-Fos and NFATc1}

Given that c-Fos and NFATC1 serve as the master contributors to osteoclastogenesis, we further evaluated the effect of Spinosin on RANKL-meidated expression of c-Fos and NFATc1. Western blot analysis showed that the expression of c-Fos and NFATc1 was enhanced by the treatment of RANKL, in which the Spinosin treatment could reverse this effect $(P<0.001$, Fig. 5).

\subsection{Spinosin inhibits OVX-induced bone loss in vivo}

To further examine the effect of Spinosin on osteoclastogenesis in vivo, the OVX-induced inflammatory bone resorption mouse model was established. The micro-CT analysis of the tibias showed that the OVX treatment stimulated the bone loss in the tibias while the treatment of Spinosin rescued the phenotype (Fig. 6A). Histomorphometric analysis revealed that the bone volume to total volume (BV/TV), trabecular 
thickness (Tb.Th), and trabecular number (Tb.N), and trabecular space (Tb.Sp) were inhibited by OVX treatment, in which Spinosin could reverse the effect $(P<0.001$, Fig. 6B). ELISA assays demonstrated that the serum levels of tumor necrosis factor-a (TNF-a) and tartrate-resistant acid phosphatase $5 \mathrm{~B}$ (TRAcp5B) was increased by OVX in the mice, but the treatment of Spinosin was able to reduce the phenotype ( $P<0.01$, Fig. $6 \mathrm{C}$ ). Moreover, Hematoxylin and Eosin (H\&E) and TRAP staining demonstrated that Spinosin was able to attenuate OVX-induced loss of femur bone and osteoclasts (Fig. 7A).

\section{Discussion}

Osteoporosis is featured as an osteoclast disorder stimulated by unnecessary osteolytic activation, affecting over two hundred million humans globally (2). Clinical anti-osteoporosis treatments principally comprise hormone-replacement therapy, denosumab, bisphosphonates, and parathyroid hormone. Simultaneously, long-term unfavorable effects, including thromboembolic disease, atypical fractures, and an enhanced risk of breast cancer, are common $(5,24)$. Furthermore, bisphosphonates and denosumab are well-known drugs that target osteoclasts, but they need to be injected (10). Therefore, the development of convenient and safe therapeutic strategies for the treatment of osteoporosis is urgent. Spinosa as a natural compound has presented anti-oxidative and anti-inflammatory potentials. In current study, we firstly identified that Spinosin induced an inhibitory effect on osteoclastogenesis of osteoporosis by repressing NF-KB through stimulating Nrf2/HO-1 signaling.

Multiple traditional natural compounds have been identified to modulate osteoporosis and osteoclastogenesis recent years. For instance, it has been reported that Kirenol represses RANKL-caused osteoclastogenesis and prserves ovariectomized-caused osteoporosis by inhibiting the Cav-1 signaling and $\mathrm{Ca}^{2+} /$ NFATc1 pathway (25). Cajaninstilbene acid decreases osteoporosis by reducing RANKL-related signaling and osteoclast formation (26). Tanshinone IIA relieves osteoclastogenesis by the inactivation of Akt and NF-kB pathway in ovariectomized mice (27). Moreover, previous studies have identified the potential biomedical function of Spinosin. It has been found that Spinosin inhibits Alzheimer's diseaserelated synaptic disorder by regulating plasmin activity (28). Spinosin is able to activate $\mathrm{Nrf} 2 / \mathrm{HO}-1$ signaling to inhibit the aggregation and production of $A \beta 1-42(20)$. In this study, we identified that Spinosin could inhibit RANKL-induced osteoclastogenesis and the expression of osteoclast-specific genes in the RANKL-treated BMMs. Spinosin was able to attenuate OVX-induced loss of femur bone and osteoclasts in vivo. These data suggest that Spinosin can inhibits osteoclastogenesis, providing a novel function of Spinosin in the modulation of osteoporosis and enriching the understanding of the role of traditional natural compounds in osteoporosis.

The pathogenesis of osteoporosis is complicated and NF-KB serves as a crucial regulator in osteoclastogenesis (25). RANKL leads to the IKBa degradation and releases NF-KB to the nuclear. Kir suppresses the osteoclast formation by reducing the nucleus accumulation of NF-kB, p65 phosphorylation and ІкBa degradation (25). Moreover, as a well-known suppressor of NF-kB, the activation of Nrf/HO-1 signaling is able to inhibit NF-KB activity (29). And the agents that stimulate $\mathrm{Nrf} / \mathrm{HO}-1$ signaling and inhibit NF-KB present the potential to relieve osteoclasts and osteoclastogenesis. 
For example, it has been reported that Hesperetin inhibits LPS-related bone loss and relieves RANKLrelated osteoclastogenesis through regulating Nrf/HO-1/NF-KB signaling (30). Lutein inhibits inflammation and oxidative stress by activating Nrf2 in the osteoporosis model (31). Glycyrrhizin represses oxidative stress and RANKL-caused osteoclastogenesis by suppressing MAPK and NF-KB and stimulating AMPK/Nrf2 signaling (32). Stilbene glycoside reduces oxidative injury by Nrf2/HO-1/NF-KB signaling in osteoblasts (33). Our mechanism investigation showed that Spinosin was able to suppress NF-KB signaling by activating Nrf2 pathway in BMMs. It consistent with previous studies that targeting $\mathrm{Nrf2/HO-1/NF-KB}$ signaling can alleviate osteoclastogenesis in osteoporosis. Our data provide the valuable information and mechanism that Nrf2/HO-1/NF-kB signaling is involved in Spinosin-exerted biomedical activities.

\section{Conclusion}

In conclusion, we discovered that Spinosin attenuated osteoclastogenesis of osteoporosis by inhibiting NF-KB through activating Nrf2/HO-1 signaling. Our finding provides new insights into the mechanism by which Spinosin modulates osteoporosis pathogenesis. Spinosin may serve as the potential candidate for the treatment of osteoporosis.

\section{Declarations}

\section{Ethical Approval and Consent to participate}

Informed consent was obtained from all individual participants included in the study. All producers were approved by Animal Ethics Committee of Linyi Central Hospital. Procedures operated in this research were completed in keeping with the standards set out in the Announcement of Helsinki and laboratory guidelines of research in China.

\section{Consent to publish}

Not applicable.

\section{Availability of supporting data}

The data that support the findings of this study are available on request from the corresponding author: * Yuna Zhang, Department of Pathology, Linyi Central Hospital, No.17 Jiankang Road, Yishui County, Linyi City, Shandong Province, 276400, PR. China.

E-mail address: YunaZhangLinyi@163.com

The data are not publicly available due to their containing information that could compromise the privacy of research participants.

\section{Competing interests}


All other authors have no conflicts of interest.

We declare that we do not have any commercial or associative interest that represents a conflict of interest in connection with the work submitted.

\section{Funding}

Not Applicable.

\section{Authors' contributions}

Bo Liu, Yuna Zhang: study concepts, literature research, clinical studies, data analysis, experimental studies, manuscript writing and review, study design, literature research, experimental studies and manuscript editing, definition of intellectual content, data acquisition and statistical analysis.

\section{Acknowledgements}

Not Applicable.

\section{References}

1. Eisa NH, Reddy SV, Elmansi AM, Kondrikova G, Kondrikov D, Shi XM, et al. Kynurenine Promotes RANKL-Induced Osteoclastogenesis In Vitro by Activating the Aryl Hydrocarbon Receptor Pathway. Int J Mol Sci. 2020;21(21).

2. Lee JM, Kim MJ, Lee SJ, Kim BG, Choi JY, Lee SM, et al. PDK2 deficiency prevents ovariectomyinduced bone loss in mice by regulating the RANKL-NFATc1 pathway during osteoclastogenesis. $J$ Bone Miner Res. 2020.

3. Compston J. Reducing the treatment gap in osteoporosis. Lancet Diabetes Endocrinol. 2020;8(1):7-9.

4. Reid IR. No more fracture trials in osteoporosis? Lancet Diabetes Endocrinol. 2020;8(8):650-1.

5. Zhu J, Zhang $\mathrm{C}$, Jia J, Wang $\mathrm{H}$, Leng $\mathrm{H}, \mathrm{Xu} \mathrm{Y}$, et al. Osteogenic effects in a rat osteoporosis model and femur defect model by simvastatin microcrystals. Ann N Y Acad Sci. 2020.

6. Martinez-Reina J, Calvo-Gallego JL, Pivonka P. Are drug holidays a safe option in treatment of osteoporosis? - Insights from an in silico mechanistic PK-PD model of denosumab treatment of postmenopausal osteoporosis. J Mech Behav Biomed Mater. 2020;113:104140.

7. Wei H, Xu Y, Wang Y, Xu L, Mo C, Li L, et al. Identification of Fibroblast Activation Protein as an Osteogenic Suppressor and Anti-osteoporosis Drug Target. Cell Rep. 2020;33(2):108252.

8. Jakob F, Genest F, Baron G, Stumpf U, Rudert M, Seefried L. [Regulation of bone metabolism in osteoporosis : novel drugs for osteoporosis in development]. Unfallchirurg. 2015;118(11):925-32.

9. Panagopoulos PK, Lambrou GI. Bone erosions in rheumatoid arthritis: recent developments in pathogenesis and therapeutic implications. J Musculoskelet Neuronal Interact. 2018;18(3):304-19. 
10. Menshawy A, Mattar O, Abdulkarim A, Kasem S, Nasreldin N, Menshawy E, et al. Denosumab versus bisphosphonates in patients with advanced cancers-related bone metastasis: systematic review and meta-analysis of randomized controlled trials. Support Care Cancer. 2018;26(4):1029-38.

11. Kirk B, Zanker J, Duque G. Osteosarcopenia: epidemiology, diagnosis, and treatment-facts and numbers. J Cachexia Sarcopenia Muscle. 2020;11(3):609-18.

12. Li X, Zhou D, Chi X, Li Q, Wang L, Lu B, et al. Entecavir combining Chinese herbal medicine for HBeAgpositive chronic hepatitis B patients: a randomized, controlled trial. Hepatol Int. 2020.

13. Shan S, Xie Y, Zhang C, Jia B, Li H, Li Z. Identification of polyphenol from Ziziphi spinosae semen against human colon cancer cells and colitis-associated colorectal cancer in mice. Food Funct. 2020;11(9):8259-72.

14. Cao JX, Zhang QY, Cui SY, Cui XY, Zhang J, Zhang YH, et al. Hypnotic effect of jujubosides from Semen Ziziphi Spinosae. J Ethnopharmacol. 2010;130(1):163-6.

15. Shang J, Chen XL, Li L, Zhang H, Yang L, Yang B, et al. Identification of the absorptive constituents and their metabolites in vivo of Ziziphi Spinosae Semen by UPLC-ESI-Q-TOF-MS/MS. Biomed Chromatogr. 2020:e4965.

16. Liu J, Zhai WM, Yang YX, Shi JL, Liu QT, Liu GL, et al. GABA and 5-HT systems are implicated in the anxiolytic-like effect of spinosin in mice. Pharmacol Biochem Behav. 2015;128:41-9.

17. Ko SY, Lee HE, Park SJ, Jeon SJ, Kim B, Gao Q, et al. Spinosin, a C-Glucosylflavone, from Zizyphus jujuba var. spinosa Ameliorates Abeta1-42 Oligomer-Induced Memory Impairment in Mice. Biomol Ther (Seoul). 2015;23(2):156-64.

18. Lee Y, Jeon SJ, Lee HE, Jung IH, Jo YW, Lee S, et al. Spinosin, a C-glycoside flavonoid, enhances cognitive performance and adult hippocampal neurogenesis in mice. Pharmacol Biochem Behav. 2016;145:9-16.

19. Xu F, He B, Xiao F, Yan T, Bi K, Jia Y, et al. Neuroprotective Effects of Spinosin on Recovery of Learning and Memory in a Mouse Model of Alzheimer's Disease. Biomol Ther (Seoul). 2019;27(1):717.

20. Zhang X, Wang J, Gong G, Ma R, Xu F, Yan T, et al. Spinosin Inhibits Abeta1-42 Production and Aggregation via Activating Nrf2/HO-1 Pathway. Biomol Ther (Seoul). 2020;28(3):259-66.

21. Tanaka Y, Mori H, Aoki T, Atsumi T, Kawahito Y, Nakayama H, et al. Analysis of bone metabolism during early stage and clinical benefits of early intervention with alendronate in patients with systemic rheumatic diseases treated with high-dose glucocorticoid: Early Dlagnosis and Treatment of OsteopoRosis in Japan (EDITOR-J) study. J Bone Miner Metab. 2016;34(6):646-54.

22. Chen K, Zhang N, Ding L, Zhang W, Hu J, Zhu S. Early intra-articular injection of alendronate reduces cartilage changes and subchondral bone loss in rat temporomandibular joints after ovariectomy. Int J Oral Maxillofac Surg. 2014;43(8):996-1004.

23. Weaver CM, Alexander DD, Boushey CJ, Dawson-Hughes B, Lappe JM, LeBoff MS, et al. Calcium plus vitamin $D$ supplementation and risk of fractures: an updated meta-analysis from the National Osteoporosis Foundation. Osteoporos Int. 2016;27(1):367-76. 
24. Covic A, Vervloet M, Massy ZA, Torres PU, Goldsmith D, Brandenburg V, et al. Bone and mineral disorders in chronic kidney disease: implications for cardiovascular health and ageing in the general population. Lancet Diabetes Endocrinol. 2018;6(4):319-31.

25. Zou B, Zheng J, Deng W, Tan Y, Jie L, Qu Y, et al. Kirenol inhibits RANKL-induced osteoclastogenesis and prevents ovariectomized-induced osteoporosis via suppressing the $\mathrm{Ca}(2+)-\mathrm{NFATc} 1$ and Cav-1 signaling pathways. Phytomedicine. 2020;80:153377.

26. Sun Y, Liu Y, He W, Wang C, Tickner J, Kuek V, et al. Cajaninstilbene acid inhibits osteoporosis through suppressing osteoclast formation and RANKL-induced signaling pathways. J Cell Physiol. 2019;234(7):11792-804.

27. Cheng L, Zhou S, Zhao Y, Sun Y, Xu Z, Yuan B, et al. Tanshinone IIA attenuates osteoclastogenesis in ovariectomized mice by inactivating NF-kB and Akt signaling pathways. Am J Transl Res. 2018;10(5):1457-68.

28. Cai M, Jung I, Kwon H, Cho E, Jeon J, Yun J, et al. Spinosin Attenuates Alzheimer's DiseaseAssociated Synaptic Dysfunction via Regulation of Plasmin Activity. Biomol Ther (Seoul). 2020;28(2):131-6.

29. Long H, Ruan J, Zhang M, Wang C, Huang Y. Gastrodin alleviates Tourette syndrome via Nrf-2/HO1/HMGB1/NF-small ka, CyrillicB pathway. J Biochem Mol Toxicol. 2019;33(10):e22389.

30. Liu H, Dong Y, Gao Y, Zhao L, Cai C, Qi D, et al. Hesperetin suppresses RANKL-induced osteoclastogenesis and ameliorates lipopolysaccharide-induced bone loss. J Cell Physiol. 2019;234(7):11009-22.

31. Li H, Huang C, Zhu J, Gao K, Fang J, Li H. Lutein Suppresses Oxidative Stress and Inflammation by Nrf2 Activation in an Osteoporosis Rat Model. Med Sci Monit. 2018;24:5071-5.

32. Li Z, Chen C, Zhu X, Li Y, Yu R, Xu W. Glycyrrhizin Suppresses RANKL-Induced Osteoclastogenesis and Oxidative Stress Through Inhibiting NF-kappaB and MAPK and Activating AMPK/Nrf2. Calcif Tissue Int. 2018;103(3):324-37.

33. Cheng J, Wang H, Zhang Z, Liang K. Stilbene glycoside protects osteoblasts against oxidative damage via Nrf2/HO-1 and NF-kappaB signaling pathways. Arch Med Sci. 2019;15(1):196-203.

\section{Figures}


(A)

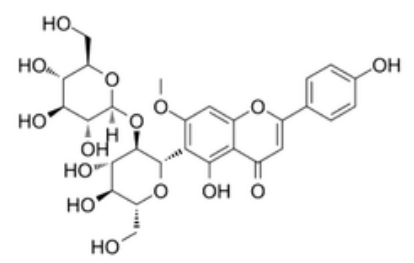

(B)
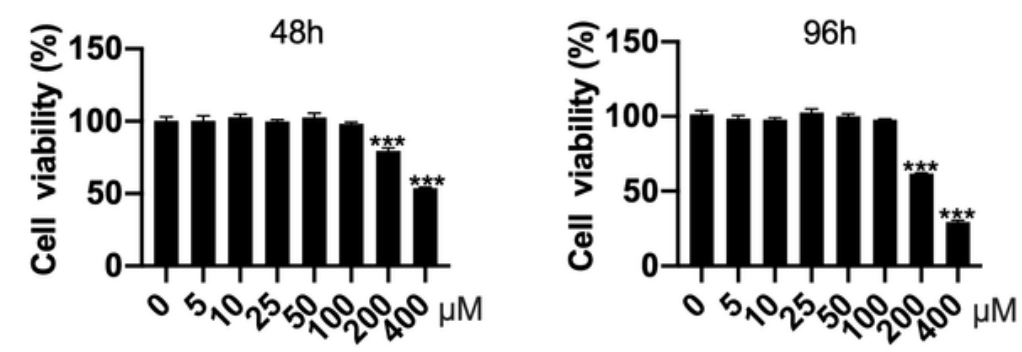

(C)

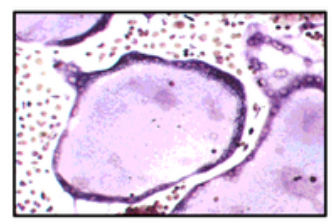

0

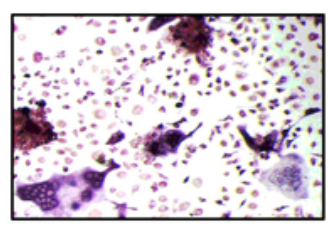

$50 \mu \mathrm{M}$

(D)

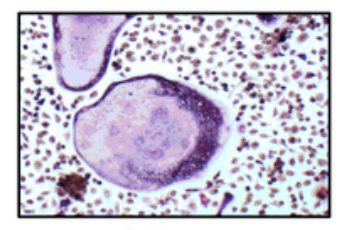

$25 \mu \mathrm{M}$
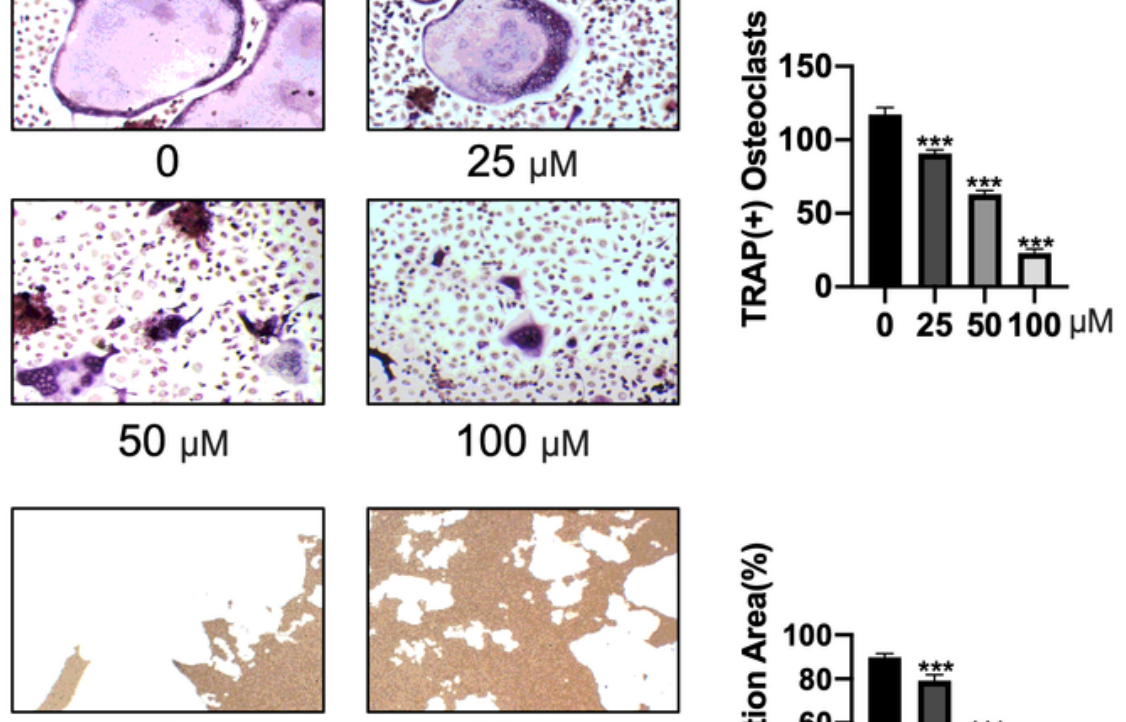

0

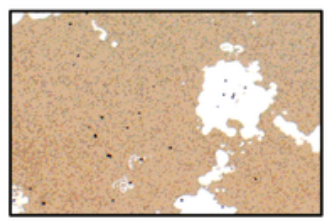

$50 \mu \mathrm{M}$

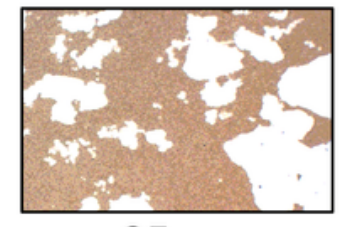

$25 \mu \mathrm{M}$

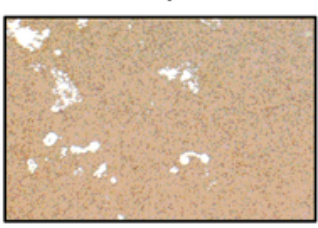

$100 \mu \mathrm{M}$

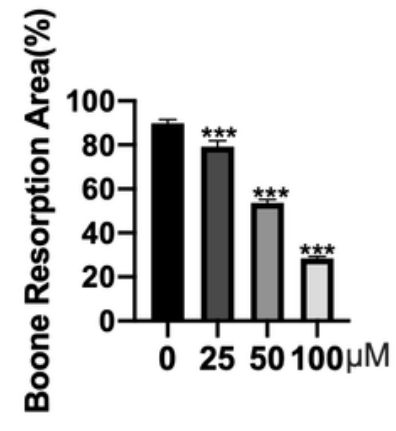

Figure 1

Spinosin inhibits RANKL-induced osteoclastogenesis. (A) The chemical structure of Spinosin was shown. (B) The cell viability was measured by CCK-8 assays in the BBMs, (C) The representative images of TRAP staining were shown in the indicated BMMs. The TRAP (+) osteoclasts were calculate. (D) The representative images of resorption pit formation assay BMMs were shown. The bone resorption area 
was calculated by the Image J software. Data are presented as mean \pm SD. Statistic significant differences were indicated: $* \star \star P<0.001$.

(A)
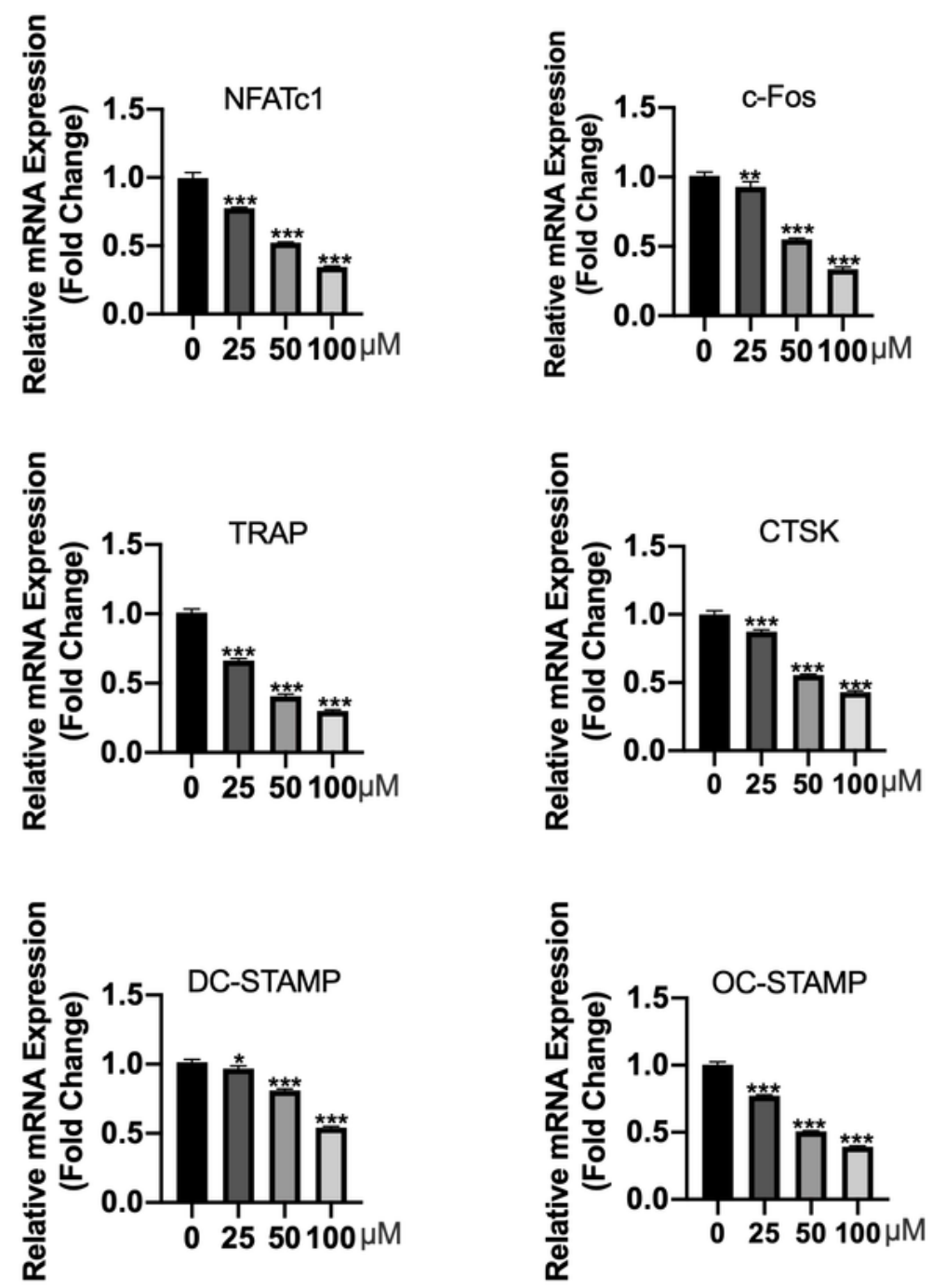

Figure 2

Spinosin reduces the expression of osteoclast-specific genes in the RANKL-treated BMMs. BMMs were treated $100 \mathrm{ng} / \mathrm{mL}$ RANKL, or co-treated with RANKL and Spinosin. The mRNA expression of NFATc1, c- 
Fos, TRAP, CTSK, DC-STAMP, and OC-STAMP was analyzed by qPCR in the BMMs. Data are presented as mean \pm SD. Statistic significant differences were indicated: $\star \star \star ~ P<0.001$.

(A)

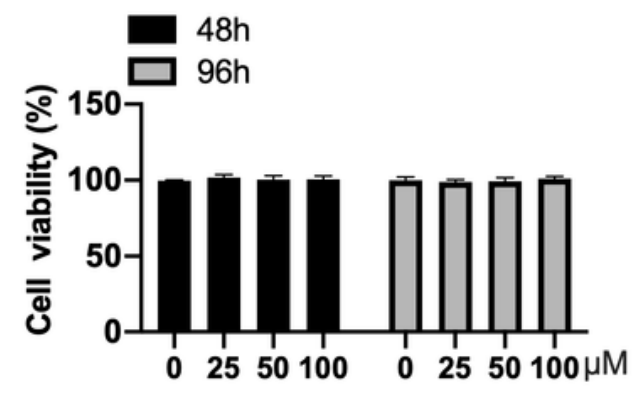

(B)

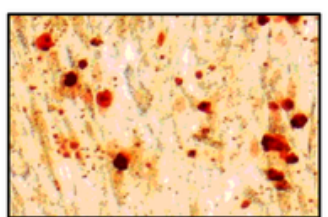

0

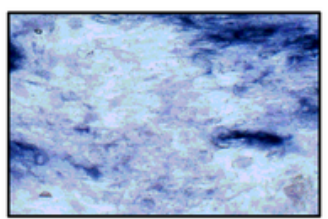

0

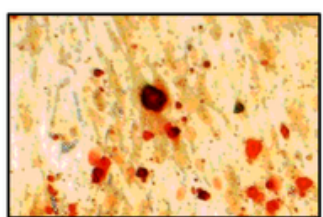

$25 \mu \mathrm{M}$

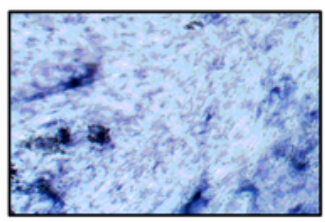

$25 \mu \mathrm{M}$

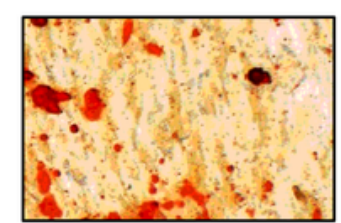

$50 \mu \mathrm{M}$

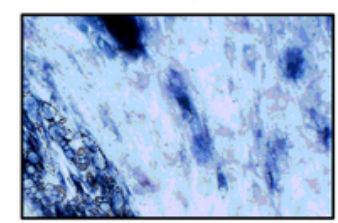

$50 \mu \mathrm{M}$

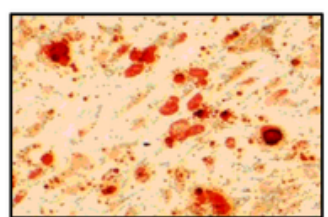

$100 \mu \mathrm{M}$

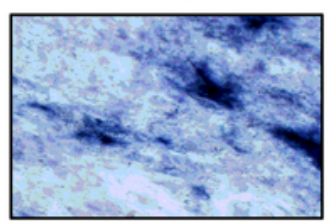

$100 \mu \mathrm{M}$

(C)
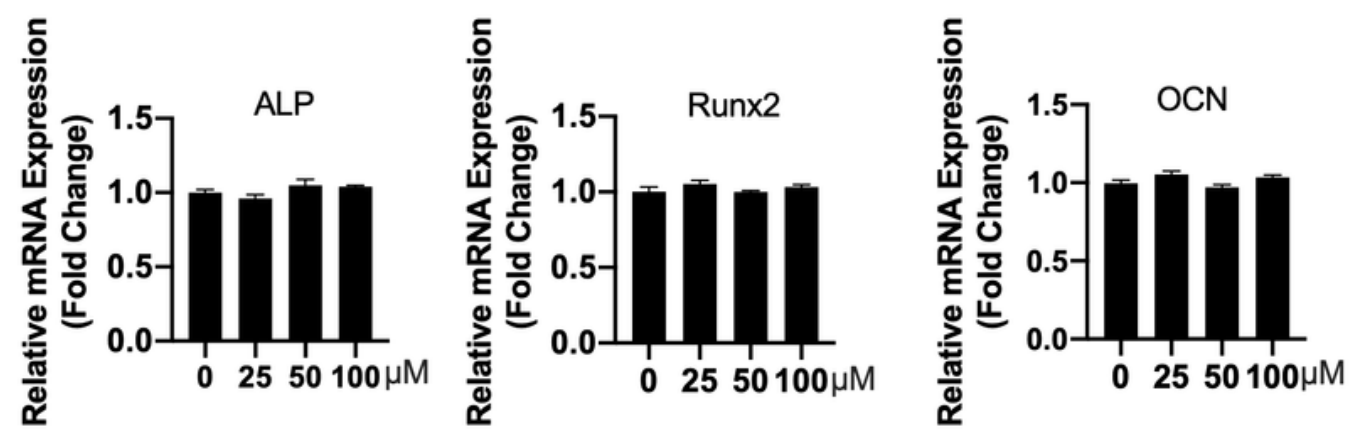

Figure 3

Spinosin fails to affect osteoblast differentiation. (A) The cell viability of MC3T3-E1 cells was measured by CCK-8 assays. (B) The osteoblastic differentiation in MC3T3-E1 cells was analyzed by ALP staining 
and Alizarin Red staining. (C) The mRNA expression of ALP1, RUNX2, and OCN was assessed by qPCR in the MC3T3-E1 cells. Data are presented as mean \pm SD.

(A)

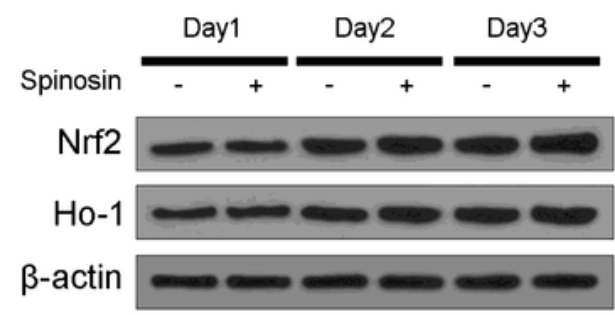

(C)

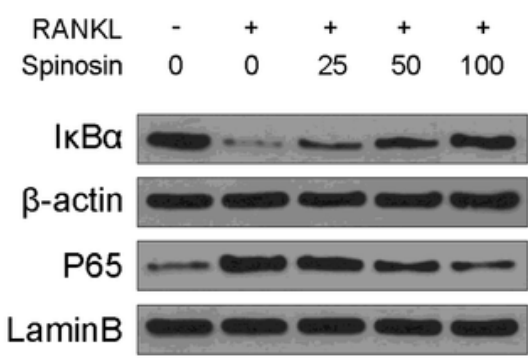

(E)

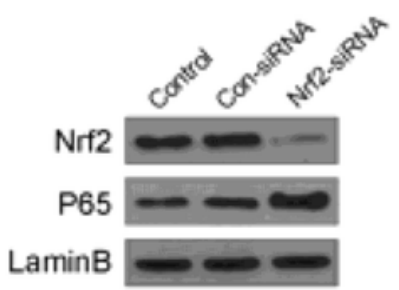

Ho-1

-actin
(B)
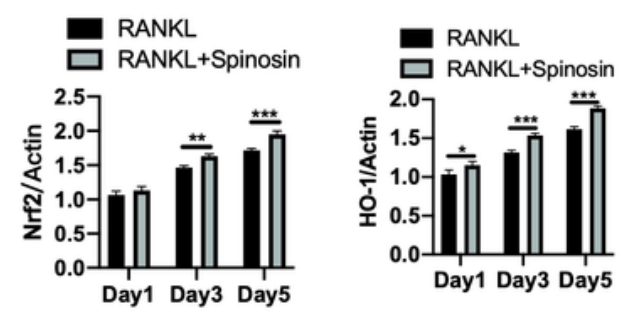

(D)
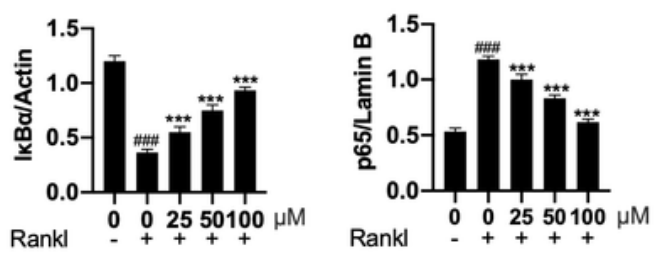

(F)
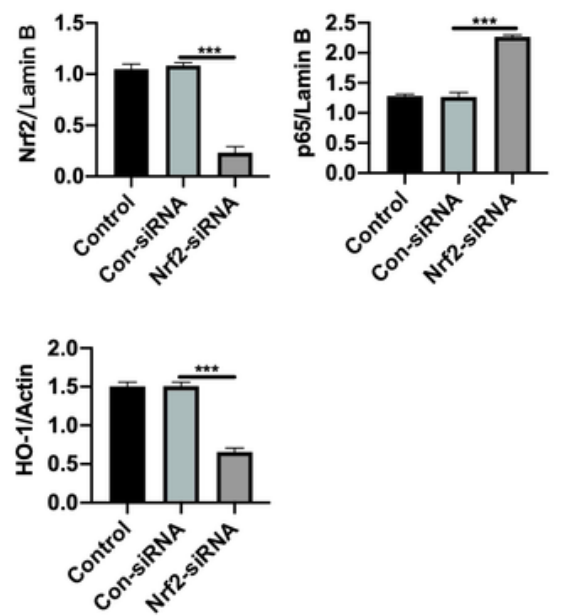

\section{Figure 4}

Spinosin suppresses NF-KB signaling by activating Nrf2 pathway. (A and B) The protein expression of $\mathrm{Nrf2}, \mathrm{HO}-1$, and $\beta$-actin was measured by Western blot analysis in the BMMs. The result of Western blot analysis was analyzed by the ImageJ software. (C and D) The protein expression of IkBa, p65, LaminB, 
and $\beta$-actin was assessed by Western blot analysis in the BMMs. The result of Western blot analysis was analyzed by the Image J software. (E and F) The protein expression of Nrf2, p65, HO-1, and $\beta$-actin was tested by Western blot analysis in the BMMs. The result of Western blot analysis was analyzed by the Image J software. Data are presented as mean \pm SD. Statistic significant differences were indicated: ** $P$ $<0.01, * \star * P<0.001$.

(A)

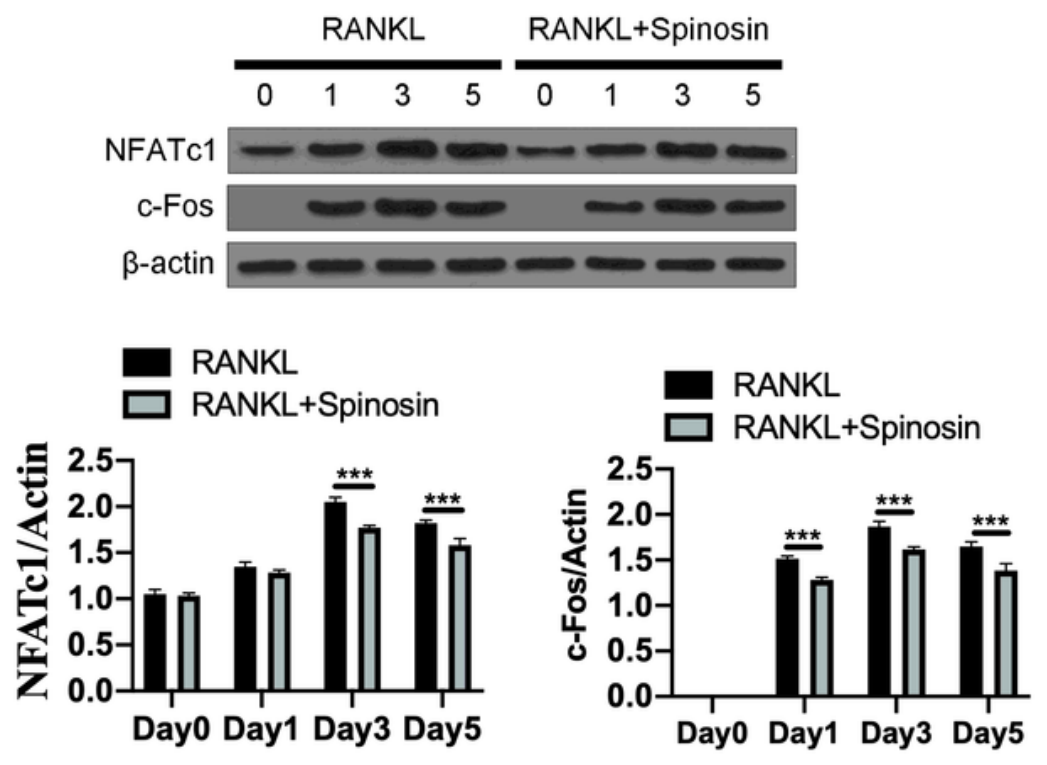

Figure 5 
Spinosin inhibits RANKL-induced expression of c-Fos and NFATc1. (A) The protein expression of NFATc1, c-Fos, and $\beta$-actin was measured by Western blot analysis in the BMMs. The result of Western blot analysis was analyzed by the ImageJ software. Data are presented as mean \pm SD. Statistic significant differences were indicated: $* \star * P<0.001$.

(A)
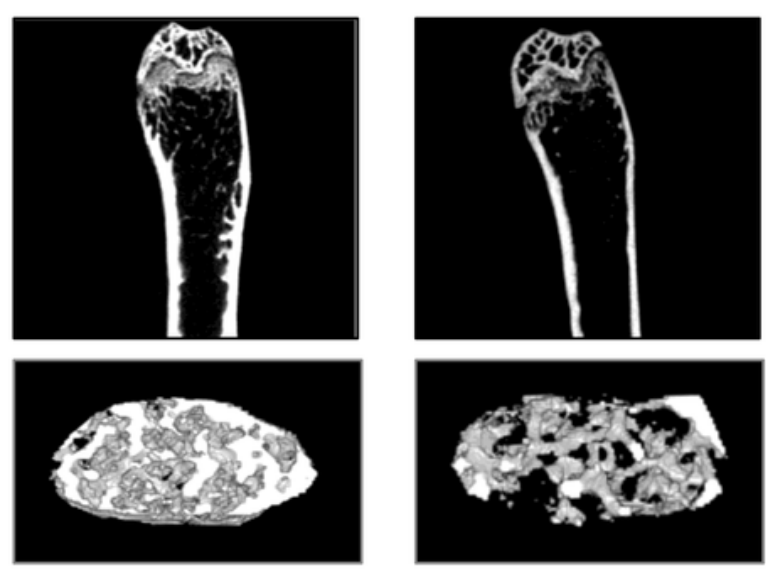

Control

(B)

$$
\text { LPS }
$$
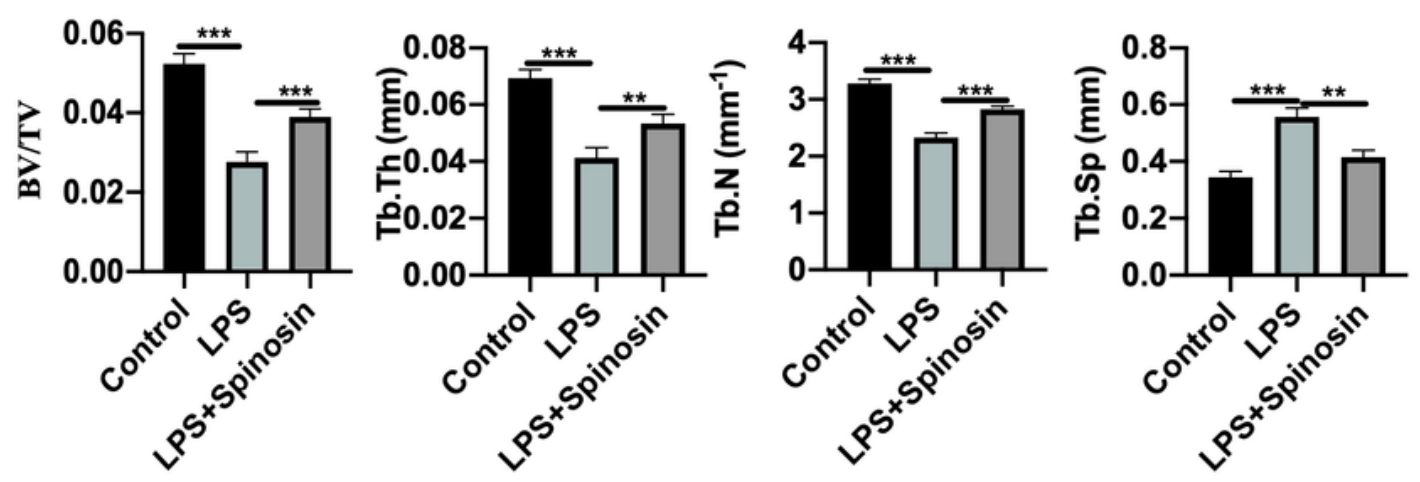

(C)
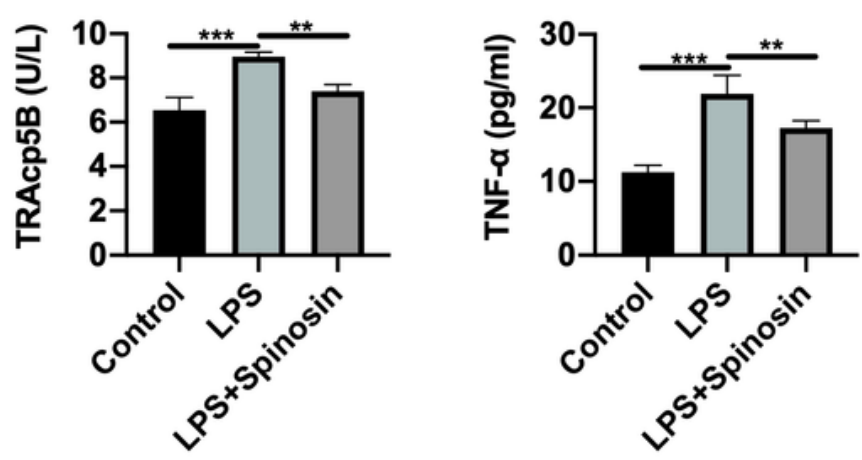

Figure 6 
Spinosin inhibits OVX-induced bone loss in vivo. (A-C) The C57BL/6 mice were treated with OVX, or cotreated with OVX and Spinosin. (A) The representative images of micro-CT analysis in the mice were shown. (B) The V/TV, Tb.Th, Tb.N, and Tb.Sp was analyzed by Histomorphometric analysis in the mice. (C) The levels of TRAcp5B and TNF-a were measure by ELISA assays. Data are presented as mean \pm SD. Statistic significant differences were indicated: $* * P<0.01$, $* \star * P<0.001$.

(A)
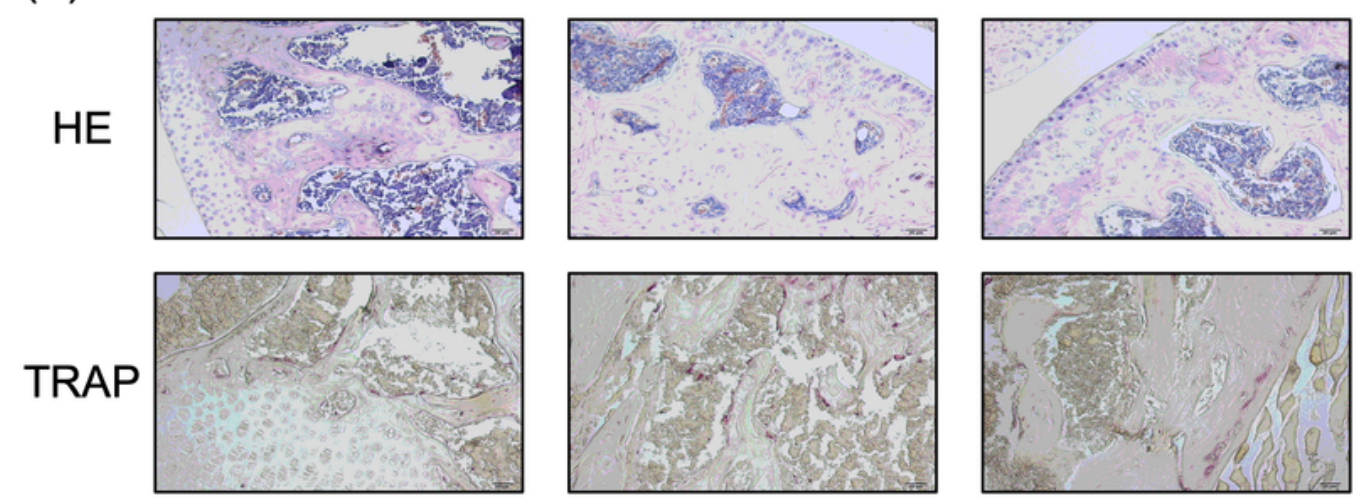

Control

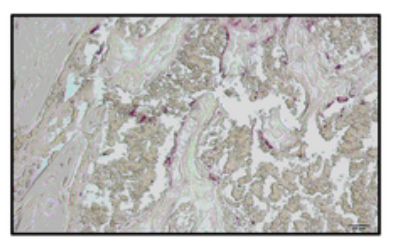

LPS

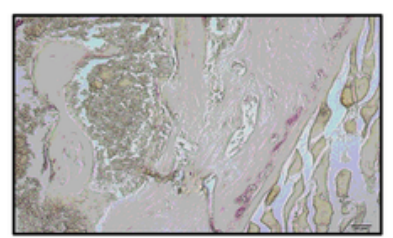

LPS+ Spinosin 
Spinosin attenuates RANKL-induced expression of c-Fos and NFATc1 femur bone in vivo. (A) The C57BL/ 6 mice were treated with OVX, or co-treated with OVX and Spinosin. The loss of femur bone and osteoclasts was analyzed by H\&E and TRAP staining. 